\title{
New Discoveries in Medicine and Physiology Originated in Urology
}

\author{
Ahmed N Ghanem* \\ Department of Urology, Egypt
}

ISSN: 2578-0379

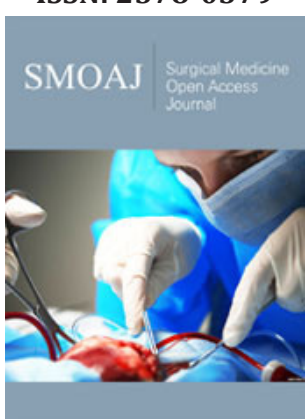

*Corresponding author: Ahmed N Ghanem, Department of Urology, Egypt

Submission: 率 January 07, 2020

Published: 趼February 20, 2020

Volume 3 - Issue 3

How to cite this article: Ahmed $\mathrm{N}$ Ghanem. New Discoveries in Medicine and Physiology Originated in Urology. Surg Med Open Acc J.3(3). SMOAJ.000564.2020. DOI: $10.31031 /$ SMOAJ.2020.03.000564.

Copyright@ Ahmed N Ghanem, This article is distributed under the terms of the Creative Commons Attribution 4.0 International License, which permits unrestricted use and redistribution provided that the original author and source are credited.

\section{Abstract}

Objective: To report new discoveries made by a single investigator working solo.

Methods: The discoveries were reported. All the investigations were self-financed done over a career life. The discoveries originated in Urology but cover specialties that include Physics, Physiology, Medicine, Nephrology, Cardiovascular, respiratory medicine and Surgery.

Result: The discoveries include 2 new vascular shocks, namely Volumetric Overload Shocks (VOS) that complicate fluid therapy in hospitals. Proving a physiological law of Starling wrong and providing the correct replacement which is the hydrodynamic of the porous orifice $(G)$ tube. Revealing many errors and misconceptions on fluid therapy and reporting its corrections. It all started with an investigation aimed at understanding a rare obscure syndrome that kills patients in Urology; the Transurethral Resection of The Prostate (TURP) syndrome. The patho-etiology of the Loin Pain Haematuria Syndrome (LPHS) was discovered revealing its link with Symptomatic Nephroptosis (SN) and reporting a new curative surgery for its treatment- the Renal Sympathetic Denervation and Nephropexy Surgery (RSD\&N). A new point of technique on the surgery for cancer bladder, namely capsule sparing cystoprostadenectomy that make the surgery easier and shorter while avoiding incontinence, impotence and urethral anastomosis was reported.

Conclusion: The discoveries started in urology but extended to Physics, Physiology, Medicine, Nephrology, Cardiovascular, respiratory medicine and Surgery. It includes 2 new shocks of VOS, proving a physiological law of Starling wrong and providing the correct replacement: the hydrodynamic of the $\mathrm{G}$ tube. It resolves the puzzles of TURP syndrome, hyponatraemia, the acute respiratory distress syndrome and LPHS.

Keywords: Shock; Hyponatraemia; The TURP syndrome; Bladder cancer surgery

Abbreviations: HN: Hyponatraemia; VOS: Volumetric Overload Shock; VOS1: Volumetric Overload Shock Type 1; VOS2: Volumetric Overload Shock Type 2; TURP: The Transurethral Resection of the Prostate; ARDS: Acute Respiratory Distress Syndrome; MVOD/F: Multiple Vital Organ Dysfunction/Failure; HST: Hypertonic Sodium Therapy; ARF: Acute Renal Failure; NaCl: Sodium chloride; NaCo3: Sodium Bicarbonate; G tube: Porous Orifice Tube; LPHS: Loin Pain Haematuria Syndrome; SN: Symptomatic Nephroptosis; RSD\&N: Renal Sympathetic Denervation and Nephropexy; IVU: Intravenous Urography; IVU-E: Intravenous Urography Erect Film; RGP: Retrograde Pyelography

\section{Introduction}

Reported here is a summary of the new discoveries in medicine and physiology originated in urology made by a single investigator working solo. All the research was self-financed without any external funds done over a career life of 32 years. The discoveries expanded to cover many specialties that include Physics, Physiology, Medicine, Nephrology, Cardiovascular, respiratory medicine and Surgery.

\section{Method}

The new discoveries were reported and are based on the following investigations: For the discovery of VOS and relation to the path-etiology of the TURP syndrome, dilution Hyponatraemia (HN) and the Acute Respiratory Distress Syndrome (ARDS) as well as phenomenon of $\mathrm{G}$ tube as replacement for the wrong Starling's law the following studies were done.

A. Physics study on the hydrodynamic of the porous orifice tube comparing it to Poiseuille's tube- based on which Starling's law attributes capillary filtration to hydrostatic pressure. 
B. Physiological study on the hind limb of sheep to determine if the capillary works as G tube not Poiseuille's tube.

C. Prospective clinical study on 100 consecutive TURP patients among whom 10 developed the TURP syndromes randomized into treatments comparing hypertonic sodium therapy (HST) with the conservative treatment of using volume expansion for treating the new shock of VOS.

D. Case series of 23 patients who suffered the TURP syndrome.

E. Critical comprehensive literature review of the literature on the capillary physiology, the TURP syndrome, ARDS and fluid therapy in hospital.

For the discovery of LPHS patho-etiology and its successful therapy the following studies were done:

A. Prospective study on 190 patients presenting with Symptomatic Nephroptosis (SN) among whom 36 (18.9\%) developed the Loin Pain Haematuria Syndrome (LPHS).

B. Critical comprehensive literature review of the literature on SN and LPHS.

\section{Result}

The physics study demonstrated that the hydrodynamic of the porous orifice $(G)$ tube is totally different from Poiseuille's tube. The side pressure in Poiseuille's tube is positive all along the entire length of the tube causing "filtration". Thus, Starling proposed the oncotic pressure of plasma proteins as opposing force to induce "absorption". The side pressure of the G tube induces pressure gradient which is negative near the inlet and turns positive near the exit. This produces a dynamic magnetic field-like fluid circulation between the G tube lumen and a surrounding fluid compartment which is autonomous in inducing both "absorption and filtration".

Literature review demonstrated that the oncotic pressure of plasma proteins does not work. The physiological evidence proves that the capillary works as G tube not Poiseuille's tube. Thus Starling' law is proved wrong on both forces. The correct replacement is the hydrodynamic of $G$ tube (Figure 1). The prospective study demonstrated that Volumetric Overload (VO) is the most significant factor in inducing the vascular shock of TURP syndrome (Figure 2; Table 1). This shock is recognized as VOS. The correct lifesaving treatment for VOS of the TURP syndrome is Hypertonic Sodium Therapy (HST) of $5 \% \mathrm{NaCl}$ or $8.4 \% \mathrm{NaCo}_{3}$.

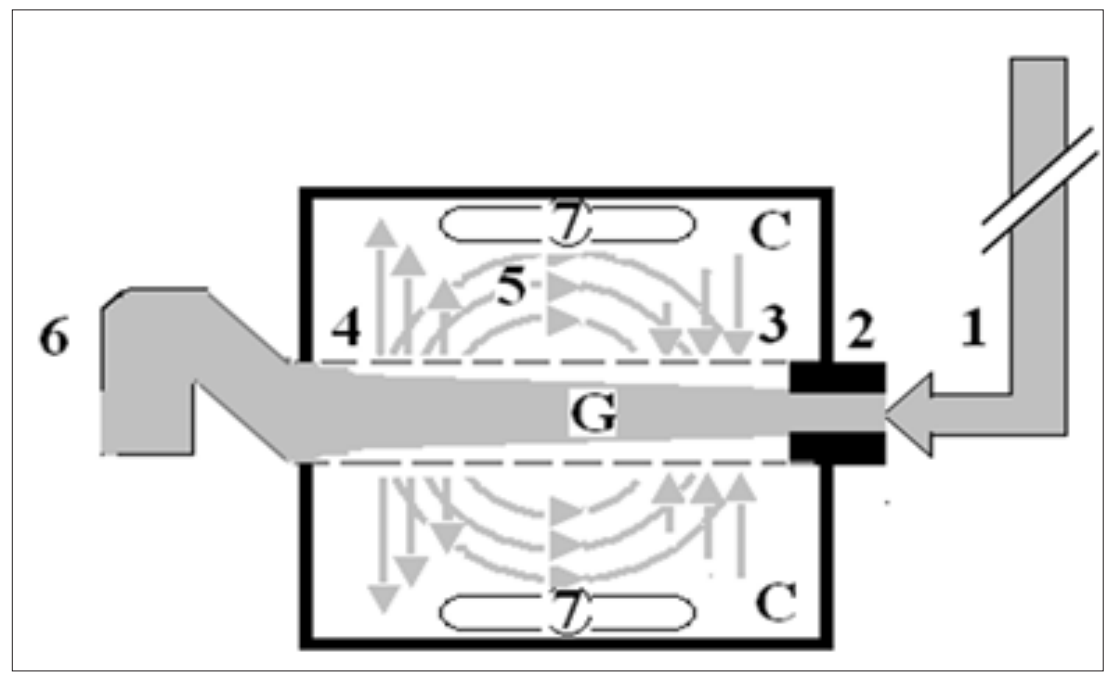

Figure 1: Shows Diagram of the porous orifice $(G)$ tube enclosed in chamber $(C)$ based on several photographs demonstrating the magnetic field-like G-C circulation phenomenon. The proximal inflow (arterial) pressure:

1. Pushes fluid through the orifice.

2. Creating fluid jet in the lumen of the $\mathrm{G}$ tube. The fluid jet creates negative side pressure gradient causing suction maximal over the proximal half of the $\mathrm{G}$ tube near the inlet.

3. That sucks fluid into lumen. The side pressure gradient turns positive pushing fluid out of lumen over the distal half maximally near the outlet.

4. Thus the fluid around $\mathrm{G}$ tube inside $\mathrm{C}$ moves in magnetic field-like fluid circulation.

5. Taking an opposite direction to lumen flow of G. tube. The inflow (arterial) pressure (1) and orifice (2) induce the negative side pressure energy creating the dynamic $\mathrm{G}-\mathrm{C}$ circulation phenomenon that is rapid, autonomous and efficient in moving fluid out from the $G$ tube lumen at (4), irrigating $C$ at (5), then sucking it back again at (3), maintaining net negative energy pressure (7) inside C. The distal outflow (venous) pressure.

6. Enhances outflow at (4) and its elevation may turn the negative energy pressure.

7. Inside $\mathrm{C}$ into positive, increasing volume and pressure inside $\mathrm{C}$ chamber. 


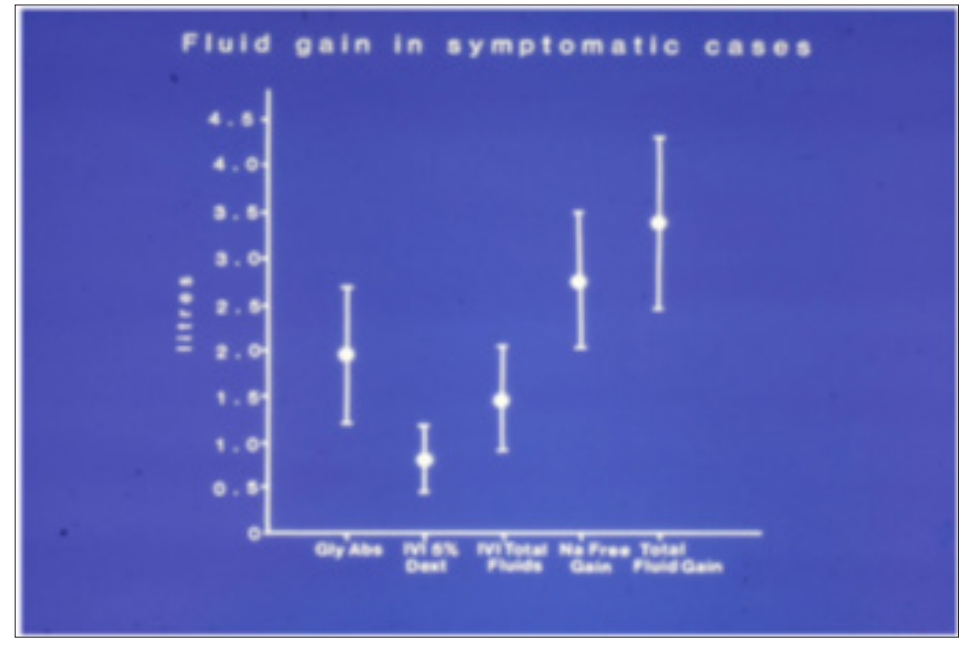

Figure 2: shows the means and standard deviations of volumetric overload in 10 symptomatic patients presenting with shock and hyponatremia among 100 consecutive patients during a prospective study on transurethral resection of the prostate. The fluids were of Glycine absorbed (Gly abs), intravenously infused 5\% Dextrose (IVI Dext) Total IVI fluids, Total Sodium-free fluid gained (Na Free Gain) and total fluid gain in liters.

Table 1: Shows the multiple regression analysis of total per-operative fluid gain, drop in measured serum osmolality (OsmM), sodium, albumin, $\mathrm{Hb}$ and increase in serum glycine occurring immediately post-operatively in relation to signs of the TURP syndrome. Volumetric gain and hypo-osmolality are the only significant factors. The significance of volumetric overload is remarkable.

\begin{tabular}{|c|c|c|c|c|c|}
\hline Parameter & Value & Std. Err & Std. Value & T Value & \\
\hline Intercept & & & 0.773 & 3.721 & 0.0001 \\
\hline Fluid Gain (I) & 0.847 & 0.228 & 1.044 & 2.42 & 0.0212 \\
\hline Osmolality & 0.033 & 0.014 & -0.375 & 0.95 & 0.0597 \\
\hline Na $^{+}$(C_B) & 0.095 & 0.049 & 0.616 & 0.4809 \\
\hline Alb (C_B) & 0.062 & 0.087 & 0.239 & 0.149 & 0.2587 \\
\hline Hb (C_B) & -0.282 & 0.246 & -0.368 & 0.4112 \\
\hline Glycine (C_B) & $-4.97 \mathrm{E}-05$ & $5.98 \mathrm{E}-05$ & -0.242 & & 0.832 \\
\hline
\end{tabular}

The case series of 23 patients demonstrated that mistaking VOS for one of the recognized shocks such as septic or hemorrhagic shock and treating it with further volumetric expansion caused death of the first 3 patients. Diagnosing VOS correctly and treating it with prompt HST saved the lives of 20 patients. VOS is of two types depending on the type of fluid inducing it. Sodium-free fluid such as $1.5 \%$ Glycine and 5\% Glucose induce VOS1. The TURP syndrome and dilution Hyponatraemia (HN) represent VOS1. Sodium-based fluid such as Normal Saline, Hartmann' solution, plasma, plasma substitute and/or blood induce VOS2. Both VOS are always mistaken for one of the recognized shocks and wrongly treated with volume expansion using isotonic sodium-based fluids causing ARDS and death. Discovery of VOS has resolved the puzzles of TURP syndrome, HN and ARDS discovering the exact patho-etiology and successful lifesaving therapy.

Discovering the link of LPHS with SN has revealed the exact patho-etology and allowed a successful curable surgery of the renal sympathetic denervation and nephropexy. The new IVU 7 sign demonstrated stretch of renal pedicle caused vessels stenosis inducing renal ischemia with medullary renal damage causing LPHS. Sympathetic neuropathy also occurs. The surgery was successfully curable in 18 patients as it addressed both issues of the patho-etiology.

\section{Discussion}

Volumetric Overload Shocks (VOS) are iatrogenic complications of fluid therapy in hospitals [1-3]. It affects urological, surgical and obstetric patients of men, women and children undergoing surgery. It is new discoveries in medicine and physiology [4] that originated in urology. The objective of this article is to bring these new discoveries into the attention of readers, particularly surgeons, anesthetists and urologists as these conditions concern them most. The scientific discoveries include 2 VOS1-3, proving the physiological law of Starling for the capillary-interstitial fluid transfer wrong and finding a new correct replacement which is the hydrodynamic of the porous orifice $(G)$ tube (Figure 1); [57]. Starling's law being wrong has resulted in many errors and misconceptions on fluid therapy [8], during prolonged surgery 
and the resuscitation of shock and the acutely ill patients. This misleads physicians into giving too much fluid which induces VOS, causing cardiac or respiratory arrest or both "cardiopulmonary arrest" immediately in theatre [9] or the acute respiratory distress syndrome (ARDS) later [10].

VOS are two types depending on the type of fluid: VOS1 is induced by sodium-free fluid such as $5 \%$ Glucose and/or $1.5 \%$ Glycine used as irrigating fluid during the Transurethral Resection of the Prostate (TURP) surgery. It is known in urology as the TURP syndrome [11] or hyponatraemic shock [12]. This VOS1 is induced by $1.5 \%$ Glycine absorption and $5 \%$ glucose infusion of about 3.5 5 liters or $>5 \%$ of body weight and is characterized with dilution Hyponatraemia (HN) [13,14]. It has 2 nadirs and 2 paradoxes15 making it dynamic and illusive16. The 2 nadirs are: The immediate drop of serum sodium level as result of dilution of the extra-cellular fluid that occurs during or immediately after surgery. The second nadir is that occurring later within 24 hours after water shift into the intracellular compartment causing spontaneous elevation of serum sodium level towards normal, yet the clinical picture gets worse due to generalized cellular edema. This cellular edema manifests as the multiple vital organ dysfunction/ failure (MVOD/F) syndrome. The 2 paradoxes are: A pathological volumetric overload induces hypotensive shock of VOS and Acute Renal Failure (ARF) which is paradoxical to the response of physiological volume replacement that treats hypotensive shock and induces diuresis [14-16].

VOS1 currently has a lifesaving therapy of Hypertonic Sodium Therapy (HST) of $5 \% \mathrm{NaCl}$ or $8.4 \% \mathrm{Co}_{3}$ [17]. It may present with cardiopulmonary arrest [9], or one or more of the other manifestations of MVOD/F syndrome- being the new name for ARDS10. The clinical manifestations include in addition to cardiorespiratory features: coma, ARF and hepatic dysfunction. It also causes coagulopathies and excessive bleeding at the surgical site. VOS1 affects women too during the trans-cervical resection of endometrium due to Glycine absorption, and during Cesarean section due to excessive 5\% Glucose infusion [14]. VOS1 initially presents with cardiovascular hypotensive shock to anesthetists and surgeons in the operating theatre characterizes not only with HN but also with bradycardia. Other dysrhythmia may occur up to cardiac arrest. Respiratory arrest or both "cardiopulmonary arrest" may occur. Survived patients of HN present to physicians by the next morning after surgery with encephalopathy coma, paralysis and convulsions.

In theatre VOS is always mistaken for one of the recognized shocks such as hemorrhagic and septic shocks then wrongly treated with further volume expansion using sodium-based isotonic fluids. This induces VOS2 and cardiopulmonary arrest that has no serum markers of $\mathrm{HN}^{2}$ and causes ARDS in patients who survive a little longer [8,9]. Multiple regression analysis has proved that volumetric overload is the most significant factor in causing the clinical picture of VOS of the TURP syndrome (Figure 2 \& 3; Table 1) [3,11]. Volumetric Overload Shock Type 2 (VOS2)13,10 is induced by massive infusion of sodium-based fluids such as normal saline, Hartmann, plasma, plasma substitutes and blood or a combination of it. VOS2 may complicate VOS1 or is induced by sodium-based fluid during fluid therapy for resuscitation of shock and the critically ill and prolonged surgery and presents with ARDS later. Volumetric gain of 12-14 liters of sodium-based fluids reported in the first article on ARDS [18], which is the only article in the whole literature, other than the articles of mine some of which are referenced here, that documents the volume of retained fluid in ARDS.

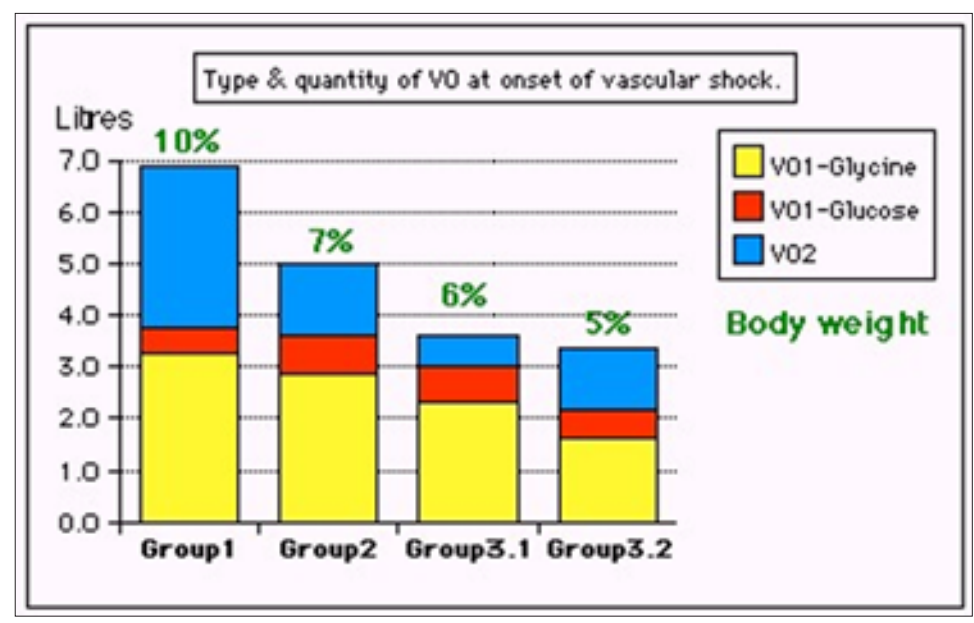

Figure 3: shows volumetric overload (VO) quantity (in litres and as percent of body weight) and types of fluids. Group 1 was the 3 patients who died in the case series as they were misdiagnosed as one of the previously known shocks and treated with further volume expansion. Group 2 were 10 patients from the series who were correctly diagnosed as volumetric overload shock and treated with Hypertonic Sodium Therapy (HST). Group 3 were 10 patients who were seen in the prospective study and subdivided into 2 groups; Group 3.1 of 5 patients treated with HST and Group 3.2 of 5 patients who were treated with guarded volume expansion using isotonic saline 
On another subject, this article [19], reports the overlooked link of Loin Pain Hematuria Syndrome (LPHS) with Symptomatic Nephroptosis (SN) and the Results of a new curative surgery; Renal Sympathetic Denervation and Nephropexy (RSD\&N) Surgery. Two new signs namely, the IVU 7 sign (Figure 4) and tube stretch hypothesis were reported demonstrating that renal pedicle stretch causing vessel stenosis, ischemia and neuropathy. This was based on a prospective study of 190 patients with SN shown on erect IVU film (IVU-E), 182 were females and 8 males. The mean age was 28.8, duration of symptoms 15.7 and hospital follow up 6.6 years. Patients showed no abnormality on IVU or ancillary imaging when supine. All patients showed renal drop of $>1.5$ vertebrae $(>5 \mathrm{~cm})$ on erect IVU film (IVU-E). Other demonstrable pathology on IVP-E film included: pelvic-ureteric junction kink affecting the right kidney in $116(61.1 \%)$ and bilateral in 19 (10\%) of patients. Stretch/ rotation of renal pedicle causing neuro-ischaemic pain of LPHS was demonstrable on the right side in 72 (37.9\%) and bilaterally in 7 patients. Complications of SN on IVU-E included both obstructive and neuro-ischaemic: obstructive complication included ballooned renal pelvis, hydronephrosis and upper pole diverticulum. Neuroischaemic complications induced by pedicle stretch and rotation/ twist were haematuria of the LPHS affecting 36 (18.9\%), auto nephropexy affecting 12 right kidneys, upper pole calyctiasis with extra-vasation affecting 28 (15.8\%) right kidney and 2 bilateral that are best shown on Retrograde Pyelography (RGP). Renal atrophy affected 4 right kidneys. Upper pole infarction affected 2 right kidneys. Retrograde pyelography also demonstrated upper pole calyctiasis with extra-vasation. Surgical treatment was used in 28 patients; 10 had simple nephropexy and 18 had RSD\&N) for severe LPHS. Four of patients treated with simple nephropexy had recurrence of LPHS while those who had RSD\&N were all cured.

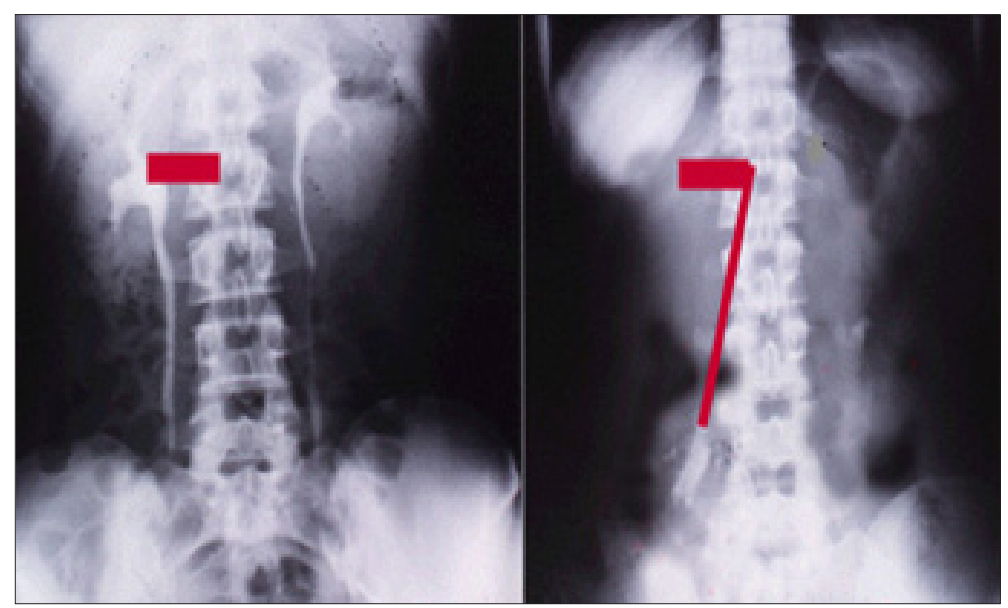

Figure 4: Shows renal pedicle mapped on a supine IVU film (Horizontal) and erect film (Vertical) limbs of 7 where the renal pedicle is stretched to 3 times its normal length, causing stenosis and ischemia.

On another subject I reported a surgical point of technique [20], for operable cancer bladder in which "capsule sparing" cystoprostadenectomy for orthotopic bladder replacement that overcomes the problems of difficult urethral anastomosis, impotence and incontinence. It makes the surgery easier and shorter. In summary, the new discoveries in medicine and physiology that originated in urology are reported. VOS are common iatrogenic complication of fluid therapy in hospitals. It may present in theatre as cardiopulmonary arrest in the operating theatre or later with coma and ARDS. VOS is 2 types: VOS1 and VOS2. VOS1 is induced by 3.5-5 liters of sodium-free fluid and is characterized with dilution HN that has 2 nadirs and 2 paradoxes, is most dynamic and illusive and currently has a lifesaving therapy of HST. VOS2 may complicate VOS1 or occur de novo complicating sodium-based fluid therapy during resuscitation of shock, acutely ill patients and prolonged surgery. It has no obvious serological markers or none. Many errors and misconceptions mislead physicians into giving too much fluid for resuscitation due to faulty rules on fluid therapy dictated by the wrong Starling's law. The correct replacement for this law is the hydrodynamic of the G tube.
The overlooked link of LPHS with SN and the Results of a new curative surgery RSD\&N were reported. Two new signs namely, the IVU 7 sign and tube stretch hypothesis demonstrating that renal pedicle stretch causing vessel stenosis, ischemia and neuropathy were reported. On another subject I reported a surgical point of technique for operable cancer bladder. Experience with "capsule sparing" cystoprostadenectomy for orthotopic bladder replacement: Overcoming the problems of impotence, incontinence and urethral anastomosis. The technique makes the surgery easier and shorter. The mentioned discoveries have resolved the puzzles of TURP syndrome, HN, ARDS and LPHS. Not only the exact pathoetiology was revealed but also a curable therapy was reported. These scientific discoveries should make the Medical World wake up, pay attention and listen to what this article has to say [21].

\section{References}

1. Ghanem AN, Ghanem SA (2016) Volumetric overload shocks: Why is starling's law for capillary interstitial fluid transfer wrong? the hydrodynamics of a porous orifice tube as alternative. Surgical Science $7(6): 245-249$. 
2. Pindoria N, Ghanem SA, Ghanem KA, Ghanem AN (2017) Volumetric overload shocks in the patho-etiology of the transurethral resection prostatectomy syndrome and acute dilution hyponatraemia. Integr Mol Med.

3. Ghanem KA, Ghanem AN (2017) Volumetric overload shocks in the patho-etiology of the transurethral resection prostatectomy syndrome and acute dilution hyponatraemia: The clinical evidence based on 23 case series. Basic Research Journal of Medicine and Clinical Sciences $6(4)$.

4. Ghanem AN (2018) Ghanem's new discoveries in medicine, physiology and urology and nephrology? Exp Tech Urol Nephrol 2(2).

5. Ghanem AN (2001) Magnetic field-like fluid circulation of a porous orifice tube and relevance to the capillary-interstitial fluid circulation: Preliminary report. Medical Hypotheses 56(3): 325-334.

6. Ghanem KA, Ghanem AN (2017) The proof and reasons that starling's law for the capillary-interstitial fluid transfer is wrong, advancing the hydrodynamics of a porous orifice $(\mathrm{G})$ tube as the real mechanism. Blood Heart and Circ 1(1): 1-7.

7. Ghanem KA, Ghanem AN (2017) The physiological proof that starling's law for the capillary-interstitial fluid transfer is wrong: Advancing the porous orifice $(\mathrm{G})$ tube phenomenon as replacement. Open Acc Res Anatomy 1(2).

8. Ghanem AN (2018) The adult respiratory distress syndrome: Volumetric overload shocks in patho-etiology, correcting errors and misconceptions on fluid therapy, vascular and capillary physiology. Surg Med Open Acc J 2(2).

9. Ghanem AN (2019) Cardiac arrest and Volumetric Overload Shocks (VOS) complicating fluid therapy. EC Clinical and Medical Case Reports.

10. Ghanem AN (2019) Volumetric Overload Shocks (VOS) causing the Acute Respiratory Distress Syndrome (ARDS). International Journal of Current Medical and Pharmaceutical Research.

11. Ghanem AN, Ward JP (1990) Osmotic and metabolic sequelae of volumetric overload in relation to the TURP syndrome. Br J Uro 66(1): 71-78
12. Harrison RH, Boren JS, Robinson JR (1956) Dilutional hyponatraemic shock: Another concept of the transurethral prostatic reaction. J Urol 75 (1): 95-110.

13. Ghanem AN (2019) Post-surgical hyponatraemia: Problems of management resolved by revealing its relation to volumetric overload shocks. EC Cardiology 6(8): 708-714.

14. Ghanem AN (2019) Postoperative dilution hyponatraemia and the TURP syndrome: Critical analytical review of literature on patho-etiology and therapy. EC Emergency Medicine and Critical Care 3(8): 507-514.

15. Ghanem AN (2018) Hyponatraemia: Nadirs and paradoxes of the missing volumetric overload. Open Access Journal of Surgery 10(1).

16. Ghanem AN, Ghanem SA, Ghanem KA, Pindoria N, Elsayed YS (2019) Illusive dynamic nadirs and masks of postoperative hyponatraemia and the TURP syndrome: Volumetric overload over time (VO/T) concept for resolving its puzzle. JOJ Uro \& Nephron 6(4)

17. Ghanem AN (2018) Therapy of hyponatremia: End of era or minority report? Biomed J Sci \& Tech Res 11(4).

18. Ashbaugh DG, Bigelow DB, Petty TL, Levine BE (1967) Acute respiratory distress in adults. Lancet 2(7511): 319-323.

19. Ghanem AN (2016) Prospective observational study on loin pain hematuria syndrome complicating symptomatic nephroptosis and the results of renal sympathetic denervation and nephropexy surgery. J J Nephro Urol 3(1): 024.

20. Ghanem AN (2002) Experience with capsule sparing cystoprostadenectomy for orthotopic bladder replacement: Overcoming the problems of impotence, incontinence and urethral anastomosis. BJU International 90(6): 617-620.

21. Ghanem AN (2019) Medical world wake up, pay attention and listen: Ghanem's new scientific discoveries in medicine, physiology, urology, nephrology, cardiovascular and surgery. EC Clinical \& Medical Case Reports 2(9): 1-6. 